Mara Rejane Barroso Barcelos ${ }^{1}$

Paulo Roberto Merçon de Vargas ${ }^{2}$

CARLA BARON ${ }^{3}$

Angélica EspinOSa Miranda ${ }^{4}$

Artigos originais

Palavras-chave

Doenças sexualmente transmissíveis/ epidemiologia Assunção de riscos

Centros de Saúde

Mulheres

Keywords

Sexually transmitted diseases/ epidemiology

Risk-taking

Health centers

Women

\title{
Infecções genitais em mulheres atendidas em Unidade Básica de Saúde: prevalência e fatores de risco
}

\author{
Genital infections in women attending a Primary Unit of Health: \\ prevalence and risk behaviors
}

\section{Resumo}

OBJETIVO: descrever a prevalência e o perfil comportamental para infecções genitais em mulheres atendidas em uma Unidade Básica de Saúde em Vitória, Espírito Santo. MÉTODOS: estudo em corte transversal, realizado em mulheres de 15 a 49 anos, atendidas numa área atendida pelo Programa Saúde da Família (PSF). Os critérios de exclusão foram: ter sido submetida a um exame ginecológico há menos de um ano e ter histórico de tratamento recente (nos últimos três meses) para infecções genitais. Foi aplicada entrevista contendo dados sócio-demográficos, clínicos e comportamentais. Espécimes genitais foram coletados para citologia, bacterioscopia pelo Gram e cultura; e amostra de urina para teste de biologia molecular para Chlamydia trachomatis. RESULTADOS: participaram do estudo 299 mulheres. A mediana de idade foi de 30,0 (intervalo interquartil: 24;38) anos; a média de idade do primeiro coito foi de 17,3 (dp=3,6) anos. A média de idade da primeira gravidez foi de 19,2 (dp=3,9) anos. Aproximadamente $70 \%$ relataram até oito anos de escolaridade; $5 \%$ relataram infecção sexualmente transmissível prévia e $8 \%$ uso de drogas ilícitas. Somente $23,7 \%$ relataram uso consistente de preservativo. As queixas clínicas relatadas foram: úlcera genital (3\%); disúria (7,7\%); fluxo vaginal $(46,6 \%)$; prurido (20\%) e dor pélvica (18\%). As taxas de prevalência foram: Chlamydia trachomatis com 7,4\%; gonorréia $2 \%$; tricomoníase $2 \%$; vaginose bacteriana $21,3 \%$; candidíase 9, $3 \%$; e alterações citológicas sugestivas de vírus 3,3\%. No modelo final de regressão logística, os fatores independentemente associados a infecções genitais foram: muco cervical anormal, $O R=9,7$ (IC95\%=5,6-13,7); realização de teste de HIV prévio, $O R=6,5$ (IC95\%=4,0-8,9); ter mais de um parceiro no último ano, OR=3,9 (IC95\%=2,7-5,0) e ter mais de um parceiro na vida, $O R=4,7$ (IC95\%=2,4-6,8). CONCLUSÕES: os resultados mostram alta freqüência de infecções genitais e a necessidade de medidas de prevenção, como o rastreamento de infecções sexualmente transmissíveis e programas de redução de risco em mulheres que procuram o serviço ginecológico de rotina.

\section{Abstract}

PURPOSE: to describe the prevalence and behavioral profile of genital infections in women attended at a Primary Health Unit in Vitoria, ES. METHODS: a transversal study including 14 to 49 -year-old women attended by the Family Health Program (FHP). Exclusion criteria were: having been submitted to gynecological examination in less than one year before, and history of recent treatment (in the last three months) for genital infections. An interview including socio-demographic, clinical and behavioral data was applied. Genital specimens were collected for cytology, GRAM bacterioscopy and culture, and urine sample for molecular biological test for Chlamydia trachomatis. RESULTS: two hundred and ninetynine women took part in the study. The median age was 30.0 (interquartile interval: $24 ; 38$ ) years old; the average age of the first intercourse was 17.3 (sd=3.6) years old. The first pregnancy average age was 19.2 (3.9) years old. About 70\% reported up to 8 years of schooling; $5 \%$ reported previous Sexually Transmitted Diseases (STD), and 8\%, the use of illicit drugs. Only 23.7\% reported consistent use of condoms. Clinical complaints were: genital ulcer (3\%); dysuria (7.7\%); vaginal discharge (46.6\%): pruritus (20\%) and pelvic pain (18\%). Prevalence rates were: Chlamydia trachomatis $7.4 \%$; gonorrhea $2 \%$; trichomoniasis $2 \%$; bacterial vaginosis $21.3 \%$; candidiasis $9.3 \%$; and cytological

Correspondência:

Mara Rejane Barroso Barcelos Núdeo de Doencas Infecciosas da Universidade Federal do Espirito Santo Avenida Marechal Campos, 1.468 - Maruípe CEP 29040-091 - Vitória/ES Fone: (27) 3335-7210 Fax: (27) $3335-7504$ E-mail: mararsb@gmail.com

Recebido

12/5/08
Núcleo de Doenças Infecciosas da Universidade Federal do Espírito Santo (UFES) e Prefeitura Munipal de Vitória- ES.

Mestre; Médica Educadora Permanente do Programa Saúde Família (PSF) da Prefeitura Municipal de Vitória (ES), Brasil.

2 Professor Adjunto do Departamento de Patologia da Universidade Federal do Espírito Santo -UFES - Vitória (ES), Brasil.

${ }^{3}$ Bióloga do Núcleo de Doenças Infecciosas da Universidade Federal do Espírito Santo - UFES -Vitória (ES), Brasil.

${ }_{4}^{4}$ Professora Adjunto do Departamento de Medicina Social da Universidade Federal do Espírito Santo - UFES - Vitória (ES), Brasil. 
changes suggestive of HPV 3.3\%. In the final logistic regression model, the factors independently associated to genital infections were: abnormal cervical mucus, $\mathrm{OR}=9.7(\mathrm{C} \mid 95 \%=5.6-13.7)$, previous HIV testing, $\mathrm{OR}=6.5(\mathrm{C} \mid 95 \%=4.0-8.9)$, having more than one partner during the previous year, $\mathrm{OR}=3.9$ $(C \mid 95 \%=2.7-5.0)$, and having more than one partner in life, $O R=4.7(C 195 \%=2.4-6.8)$.CONCLUSIONS: results show a high rate of genital infections and the need of preventive measures, such as STD surveys and risk reduction programs for women that look for routine gynecological service.

\section{Introdução}

As infecções do trato reprodutivo (ITR), incluindo infecções sexualmente transmissíveis (IST), merecem atenção especial da saúde pública. As IST estão entre as primeiras cinco categorias de doenças para as quais adultos em países em desenvolvimento buscam ajuda médica ${ }^{1}$; geralmente, elas causam desconforto e perda de produtividade econômica ${ }^{2}$. As seqüelas mais sérias e de maior duração surgem nas mulheres: doença inflamatória pélvica, câncer cervical, infertilidade, aborto espontâneo e gravidez ectópica, que pode levar ao óbito materno ${ }^{1,3}$. A presença de uma IST aumenta de três a cinco vezes os riscos de se adquirir e transmitir a infecção por $\mathrm{HIV}^{4,5}$, e a vaginose bacteriana pode ser um co-fator de transmissão do HIV, principalmente entre mulheres jovens ${ }^{6}$.

O manuseio efetivo das infecções genitais é importante para o controle de IST e ITR, uma vez que ele previne o desenvolvimento de complicações e seqüelas, diminui o avanço dessas infecções na comunidade e oferece uma oportunidade única para uma educação direcionada sobre a prevenção do $\mathrm{HIV}^{4}$. O tratamento adequado dessas infecções num primeiro contato entre pacientes e profissionais de saúde é, portanto, uma importante medida de saúde pública. No caso das mulheres, há o potencial de se influenciar o futuro comportamento sexual e o hábito de buscar tratamento em um estágio inicial da história natural da doença ${ }^{1}$. Dados epidemiológicos confiáveis de mulheres brasileiras com IST e outras ITR, assim como vaginose bacteriana e candidíase, são escassos ${ }^{7}$.

O Ministério da Saúde iniciou a implantação do Programa de Saúde da Família (PSF) na década de 1990. O principal propósito do PSF é reorganizar a prática da atenção à saúde em novas bases e substituir o modelo tradicional, levando a assistência à saúde para mais perto da família, e melhorando a qualidade de vida das pessoas ${ }^{8}$. A ausência de dados precisos sobre o número de casos de DST e sobre os padrões de comportamento das mulheres que buscam atendimento no PSF em Vitória motivou a realização desse estudo para o conhecimento da realidade local e o planejamento de estratégias de intervenção e prevenção para esta população. O principal objetivo deste estudo foi descrever as taxas de prevalência e o perfil clínico e comportamental para infecções genitais em mulheres atendidas em uma unidade primária do PSF.

\section{Métodos}

Foi realizado um estudo em corte transversal numa unidade do PSF em Vitória, Espírito Santo, Brasil, no período de agosto de 2003 a março de 2004.

A população de referência foi de mulheres cadastradas para atendimento na Unidade Básica de Saúde do bairro Consolação em Vitória. Foram incluídas no estudo, mulheres na faixa etária de 15 a 49 anos, que já tinham iniciado a atividade sexual, buscado atendimento na unidade de saúde e concordado em participar do estudo. Os critérios de exclusão foram os seguintes, ter sido submetida a exame ginecológico há menos de um ano e ter histórico de tratamento recente (nos últimos três meses) para infecções genitais.

O cálculo do tamanho da amostra foi feito considerando a prevalência de infecção pela Chlamydia trachomatis de aproximadamente $5 \%$. Com base neste cálculo, o estudo buscou incluir 292 mulheres; considerando uma perda de $10 \%$ e o número final necessário seria de 321 participantes.

O Conselho de Ética em Pesquisa do Centro de Ciências da Saúde da Universidade Federal do Espírito Santo (UFES), Vitória (ES), aprovou o protocolo de pesquisa. Foi obtido consentimento informado, por escrito, de todos os participantes, de acordo com as determinações legais no Brasil. Foi oferecido tratamento de acordo com o manual de controle das DST do Ministério da Saúde a todas as mulheres com infecções genitais.

O questionário empregado foi constituído pelo registro de dados pessoais (nome, data de nascimento, naturalidade, profissão, cor e estado civil), dados clínicos (inventário de sintomas e de uso de medicamentos, histórico de doenças crônicas, cirurgias, infecções sexualmente transmissíveis, transfusão sanguínea, uso de drogas, alergias e internações prévias), história ginecológica (menarca, coitarca, data do último coito, data da última menstruação, padrão do ciclo menstrual, paridade, número de parceiros, freqüência da atividade sexual, uso de métodos anticoncepcionais), descrição do exame clínico e dos resultados dos exames laboratoriais. A entrevista foi seguida de exames físico e pélvico, durante os quais, amostras cérvico-vaginais foram coletadas com swabs de forma rotineira.

Os seguintes testes foram realizados: preparação citológica de fluxo vaginal para tricomoníase; bacterioscopia pelo Gram de espécimes da vagina e colo do 
útero para amostras celulares de vaginose bacteriana e gonorréia, respectivamente; citologia endo e ectocervical classificada de acordo com o protocolo de Bethesda $2001{ }^{10}$. A citologia foi usada também para avaliação de características de infecção pelo vírus do papiloma humano (HPV) - as mudanças citológicas do HPV foram presumidas pela presença de atipias coilocitóticas e epitélio escamoso do colo do útero. Foi feita cultura para Neisseria gonorrhoeae e o material foi coletado com swab de algodão da endocérvice e incubado em Ágar Thayer-Martin modificado por 24 horas a $37^{\circ} \mathrm{C}$ numa atmosfera de $5 \%$ de dióxido de carbono. Foi também efetuada a reação em cadeia da polimerase (PCR - laboratórios Roche) para infecção por Chlamydia trachomatis nas amostras de urina.

A vaginose bacteriana foi diagnosticada baseada na presença de pelo menos três dos quatro sinais dos Critérios de Amsel et al. ${ }^{11}$ : a) fluxo vaginal viscoso branco e homogêneo característico; b) fluido vaginal com $\mathrm{pH}>4,5$; c) liberação de odor de peixe vindo do fluído vaginal quando misturado com $\mathrm{KOH} 10 \%$; e d) clue cells compondo pelo menos $20 \%$ das células epiteliais vaginais, confirmado pelo Gram.

\section{Análise dos dados}

Foi realizada análise estatística descritiva, incluindo distribuições de freqüência de dados categóricos e cálculo de médias e desvios padrão para variáveis contínuas. A prevalência foi calculada para refletir a freqüência relativa de infecções genitais, com intervalos de confiança (IC) de 95\%. Odd ratios e IC $95 \%$ foram calculados em análise bivariada para estimar o poder de associação entre a presença de infecção genital e cada comportamento de risco em potencial. As variáveis que foram significativas em $\mathrm{p}<0,15$ na análise bivariada e variáveis adicionais baseadas em associações conhecidas a priori (por exemplo, idade e número de parceiros sexuais) foram consideradas na análise multivariada. Os principais fatores de risco foram avaliados gradualmente por meio de um modelo de regressão logística, com $15 \%$ como o valor de p crítico para a entrada de variáveis e $10 \%$ como critério para a eliminação de variáveis.

\section{Resultados}

Um total de 299 mulheres (93,1\% das convidadas) aceitou participar do estudo. A média de idade foi de 30,0 (intervalo interquartil: 24;38) anos de idade. Cerca de $70 \%$ delas tinham mais de oito anos de escolaridade. Em relação às atividades profissionais, $35,7 \%$ eram donas de casa e $31,7 \%$, empregadas domésticas. Mais de $60 \%$ eram casadas ou tinham um parceiro estável.
Tabela 1 - Prevalência de infecções genitais entre 299 mulheres atendidas em uma unidade Primária de Saúde em Vitória, ES

\begin{tabular}{|c|c|c|c|}
\hline Infecções cérvico-vaginais e agentes etiológicos & $n$ & $\%$ & IC95\% \\
\hline Chlamydia trachomatis & 22 & 7,4 & $4,4-10,4$ \\
\hline Neisseria gonorrhoeae & 6 & 2,0 & $0,5-3,5$ \\
\hline Trichomonas vaginalis & 6 & 2,0 & $0,5-3,5$ \\
\hline Vaginose bacteriana & 64 & 21,3 & $16,7-25,9$ \\
\hline Candida albicans & 28 & 9,3 & $6,1-12,5$ \\
\hline Infecção por HPV* & 10 & 3,3 & $1,3-5,3$ \\
\hline
\end{tabular}

IC95\%=intervalo de confiança de $95 \%$.

*Achados citológicos relacionadas ao HPV.

As infecções genitais foram usadas como marcadores para a presença de associação entre comportamentos de risco e IST, que foram consideradas como presente, quando os testes de Chlamydia trachomatis e/ou Neisseria gonorrboeae e/ou Trichomonas vaginalis foram positivos. Pelo menos uma dessas três infecções estava presente em 29 mulheres $[9,7 \%$ (IC95\%=6,3-13,1\%) $]$. As taxas de prevalência das infecções e os respectivos intervalos de confiança estão descritos na Tabela 1 .

A média de idade da primeira relação sexual foi de $17,3(\mathrm{dp}=3,6)$ anos, e $37(12,3 \%)$ mulheres tiveram sua primeira relação antes dos 15 anos. A média de idade da primeira gravidez foi de 19,2 ( $\mathrm{dp}=3,9)$ anos. Nenhuma das participantes estava grávida durante a entrevista. A violência sexual e doméstica foi reportada pelas participantes do estudo em 13,7 e 4,7\%, respectivamente. O uso abusivo de álcool, ou seja, consumo de três ou mais doses por dia, $\mathrm{OR}=2,9$ (IC95\%=1,31-6,37), uso inconsistente de preservativo (raramente ou nunca), $\mathrm{OR}=2,5$ (IC95\%=1,15-5,61), e o fato de já terem realizado o teste $\mathrm{HIV}, \mathrm{OR}=2,8$ (IC95\%=1,25-6,46) foram associados à presença de infecções genitais. Ter somente um parceiro na vida, OR=0,2 (IC95\% = 0,06$0,64)$, e ter um único parceiro no último ano, $\mathrm{OR}=0,3$ (IC95\% =0,11-0,75) foram considerados fatores de proteção na análise bivariada. Os resultados estão apresentados na Tabela 2.

Considerando a presença de sinais e sintomas, um risco aumentado de infecções genitais em mulheres com teste de $\mathrm{KOH}$ positivo, $\mathrm{OR}=3,5$ (IC95\%=1,57-7,64), muco cervical turvo ao exame, $\mathrm{OR}=5,4$ (IC95\%=1,81-15,79), e $\mathrm{pH}$ vaginal anormal, OR=5,6 (IC95\%=2,08-15,03), foi observado (Tabela 3).

No modelo final de regressão logística, os fatores independentemente associados a infecções genitais foram: muco cervical anormal, OR=9,7 (IC95\% $=5,6-13,7)$, realização de teste de HIV prévio, OR=6,5 (IC95\%=4,0$8,9)$, ter mais de um parceiro no último ano, $\mathrm{OR}=3,9$ (IC95\% $=2,7-5,0)$, e ter mais de um parceiro na vida, $\mathrm{OR}=4,7(\mathrm{IC} 95 \%=2,4-6,8)$. 
Tabela 2 -Comportamento de risco e sua associação com as IST em 299 mulheres atendidas em uma unidade do Programa de Saúde da Família, em Vitória, ES

\begin{tabular}{|c|c|c|c|}
\hline \multirow[t]{2}{*}{ Comportamentos de risco } & \multicolumn{2}{|c|}{ Infecção genital } & \multirow[t]{2}{*}{ OR (IC95\%) } \\
\hline & $\begin{array}{c}\text { Presente } \\
(\%)\end{array}$ & Ausente (\%) & \\
\hline Idade de 15 a 19 anos & 11,1 & 88,9 & $1,2(0,3-4,2)$ \\
\hline$>19$ anos & 9,5 & 90,5 & 1,0 \\
\hline \multicolumn{4}{|l|}{ Consumo abusivo de álcool } \\
\hline Sim & 15,5 & 84,5 & $2,9(1,3-6,3)$ \\
\hline Não & 6,0 & 94,0 & 1,0 \\
\hline \multicolumn{4}{|l|}{ Uso de drogas ilicitas } \\
\hline Sim & 12,5 & 87,5 & $1,4(0,38-4,92)$ \\
\hline Não & 9,4 & 90,6 & 1,0 \\
\hline \multicolumn{4}{|l|}{ Primeira relação sexual } \\
\hline Antes de 15 anos & 10,9 & 89,1 & $1,2(0,56-2,71)$ \\
\hline Após 15 anos & 9,0 & 91,0 & 1,0 \\
\hline \multicolumn{4}{|l|}{ Uso de preservativo } \\
\hline Raramente/nunca & 7,4 & 92,6 & $2,5(1,15-5,61)$ \\
\hline Uso consistente & 16,9 & 83,1 & 1,0 \\
\hline Um parceiro no ano anterior & 8,1 & 9,9 & $0,29(0,11-0,75)$ \\
\hline $\begin{array}{l}\text { Mais de um parceiro no ano } \\
\text { anterior }\end{array}$ & 23,3 & 76,7 & 1,0 \\
\hline Um parceiro na vida & 2,8 & 97,2 & $0,19(0,06-0,64)$ \\
\hline Mais de um parceiro na vida & 13,4 & 86,6 & 1,0 \\
\hline \multicolumn{4}{|l|}{ IST prévia } \\
\hline Sim & 13,3 & 86,7 & $1,5(0,32-6,86)$ \\
\hline Não & 9,5 & 90,5 & 1,0 \\
\hline \multicolumn{4}{|l|}{ Papanicolaou prévio: } \\
\hline$>3$ anos & 11,3 & 88,8 & $1,3(0,55-2,91)$ \\
\hline$<3$ anos & 9,1 & 90,9 & 1,0 \\
\hline \multicolumn{4}{|l|}{ Teste de HIV prévio: } \\
\hline Sim & 14,4 & 85,6 & $2,8(1,25-6,46)$ \\
\hline Não & 5,6 & 94,4 & 1,0 \\
\hline
\end{tabular}

OR=odds ratio; IC95\%=intervalo de confiança de $95 \%$.

\section{Discussão}

Este estudo sobre IST em mulheres atendidas em uma Unidade Básica de Saúde em Vitória, procurou investigar a infecção por clamídia, gonorréia e tricomoníase e os riscos relacionados a essas infecções. Foi demonstrada uma alta prevalência de pelo menos uma dessas infecções nessa população $(9,7 \%)$. As IST estavam significativamente associadas ao número de parceiros sexuais, ao muco cervical anormal e a ter realizado teste prévio de HIV.

A prevalência de infecção por clamídia relatada neste estudo mostra uma taxa em concordância com outros estudos realizados no Brasil, mostrando números que vão de 4,7 a $12,2 \%^{12-16}$. A taxa predominante de gonorréia foi menor (2\%) que a de clamídia, mas está de acordo com outros estudos brasileiros ${ }^{15,17}$. Nas últimas décadas, observa-se uma diminuição no número de casos de tricomoníase. Isso pode ser explicado pelo tratamento com metronidazol e melhores condições de saúde da população ${ }^{18,19}$. Outros
Tabela 3 - Aspectos clínicos e suas associaç̃oes com as IST em 299 mulheres sendo atendidas em uma unidade do Programa de Saúde da Família, em Vitória, ES

\begin{tabular}{|c|c|c|c|}
\hline \multirow[t]{2}{*}{ Aspectos clínicos } & \multicolumn{2}{|c|}{ Infecção genital } & \multirow[t]{2}{*}{ OR (IC95\%) } \\
\hline & Presente (\%) & Ausente (\%) & \\
\hline \multicolumn{4}{|c|}{ Queixa de fluxo vaginal } \\
\hline Sim & 11,0 & 89,0 & $1,2(0,53-2,80)$ \\
\hline Não & 9,2 & 90,8 & 1,0 \\
\hline \multicolumn{4}{|l|}{ Queixa de disúria } \\
\hline Sim & 13,0 & 87,0 & $1,4(0,40-5,20)$ \\
\hline Não & 9,4 & 90,6 & 1,0 \\
\hline \multicolumn{4}{|l|}{ Queixa de prurido } \\
\hline Sim & 15,0 & 85,0 & $1,9(0,84-4,51)$ \\
\hline Não & 8,3 & 91,7 & 1,0 \\
\hline \multicolumn{4}{|l|}{ Dor pélvica } \\
\hline Sim & 7,4 & 92,6 & $0,7(0,24-2,12)$ \\
\hline Não & 10,2 & 89,8 & 1,0 \\
\hline \multicolumn{4}{|l|}{ Muco cervical } \\
\hline Normal & 14,6 & 85,4 & $5,4(1,81-15,79)$ \\
\hline Anormal & 3,1 & 96,9 & 1,0 \\
\hline \multicolumn{4}{|l|}{ Fluxo vaginal } \\
\hline Normal & 10,0 & 90,0 & $1,0(0,42-3,85)$ \\
\hline Anormal & 8,0 & 92,0 & 1,0 \\
\hline \multicolumn{4}{|l|}{ Ectrópio cervical } \\
\hline Sim & 11,1 & 88,9 & $1,3(0,62-2,85)$ \\
\hline Não & 8,6 & 91,4 & 1,0 \\
\hline \multicolumn{4}{|l|}{ Teste de КОН } \\
\hline Positivo & 17,1 & 82,9 & $35(1,57-7,64)$ \\
\hline Negativo & 5,6 & 94,4 & 1,0 \\
\hline \multicolumn{4}{|l|}{ Teste de Schiller } \\
\hline Positivo & 11,2 & 88,8 & $1,4(0,65-3,01)$ \\
\hline Negativo & 8,3 & 91,7 & 1,0 \\
\hline \multicolumn{4}{|l|}{ pH vaginal } \\
\hline Normal & 16,1 & 83,9 & $5,6(2,08-15,03)$ \\
\hline Anormal & 3,3 & 96,7 & 1,0 \\
\hline
\end{tabular}

OR=Odds ratio; IC95\%=intervalo de confiança de $95 \%$.

estudos no Brasil mostraram taxas de cerca de $4 \%$ de tricomoníase em mulheres ${ }^{15,20}$.

Apesar de a vaginose bacteriana e a candidíase não serem consideradas IST, elas foram incluídas no estudo porque são motivos freqüentes de procura por cuidados ginecológicos ${ }^{6}$. Considerando que foi coletada a citologia cérvico-vaginal de todas as mulheres, 3,3\% de alterações citológicas que sugerem infecção HPV foram observadas. Esses dados estão de acordo com outros estudos feitos no Brasil usando o método de Papanicolaou como métodos de detecção de HPV: $1,1^{21}$ e 2,2\% ${ }^{22}$. Em estudos usando PCR, os autores encontraram $16^{23}$ e $18,3 \%{ }^{24}$ entre as mulheres atendidas em clínicas ginecológicas públicas. Em estudo, no qual se utilizou a captura híbrida II, para HPV de alto risco, observou-se prevalência de 17,8\%, sendo que nesse método houve positividade para HPV em $14,9 \%$ das citologias normais ${ }^{25}$.

No presente estudo, as infecções genitais foram associadas ao número de parceiros sexuais, muco cervical anormal e haver 
realizado teste prévio para infecção por HIV. A associação entre infecção genital e realização de teste prévio de HIV pode ser explicada pelo fato de que pessoas que acham estar em maior risco podem procurar voluntariamente programas de aconselhamento e rastreamento da infecção pelo $\mathrm{HIV}^{26,27}$. Em relação ao número de parceiros sexuais, $84,7 \%$ das mulheres informaram ter parceiros estáveis e 46,3\% disseram nunca ter usado preservativo com seus parceiros. O número de parceiros sexuais foi inversamente proporcional ao uso regular de preservativo, pois as mulheres tendem a se sentirem seguras quando estão em um relacionamento estável e deixam de usar preservativo ${ }^{28,29}$. Em um universo de $71 \%$ das mulheres com parceiros fixos, $55,4 \%$ relataram nunca ter usado preservativo ${ }^{15}$.

Um estudo realizado em Salvador,BA, identificou a associação entre abuso de álcool e maconha com a última relação sexual sem preservativo e IST ${ }^{14}$. A alta taxa de não uso de preservativo mostra a necessidade de campanhas educacionais sobre comportamento sexual e uso do preservativo, direcionada a mulheres com parceiros estáveis.

Neste estudo, as infecções genitais não foram associadas a sintomas ginecológicos, e fluxo vaginal foi um sintoma freqüente. A precisão do diagnóstico clínico de infecções genitais é baixa, enquanto que os métodos de diagnóstico laboratorial são complexos e caros, o que pode atrasar o início do tratamento ${ }^{30}$. Nos casos em que os testes que identificam clamídia e gonorréia não estão disponíveis, a utilização da abordagem sindrômica pode ser uma ferramenta usada para diagnosticar pacientes sintomáticos ${ }^{31}$, já que há tratamento para a maioria dos organismos responsáveis pela síndrome ${ }^{32}$. Com o intuito de diminuir o número de mulheres que seriam tratadas por infecções no colo do útero desnecessariamente, uma avaliação de risco foi incorporada à abordagem sindrômica do corrimento vaginal. Como resultado, mulheres com queixa de corrimento vaginal são tratadas sistematicamente para infecções vaginais, mas somente se a sua avaliação de risco for positiva, ela também receberá tratamento para infecção por gonococos e clamídia ${ }^{33}$. O estabelecimento e o uso de protocolos nacionais para o tratamento de IST podem ajudar a assegurar que pacientes assintomáticos e sintomáticos recebam assistência e tratamento adequados em todos os níveis dos serviços de saúde. Tais protocolos podem também facilitar o treinamento e a supervisão de profissionais de saúde e podem ajudar a reduzir o risco de desenvolvimento de resistência a antimicrobianos ${ }^{31}$.

A violência doméstica e sexual foi um aspecto importante observado neste estudo. Apesar de não estar associado às infecções genitais, foi uma questão freqüentemente mencionada pelas participantes. Também foram relatados históricos de violência sexual em estudo realizado por Martins et al..$^{15} \mathrm{em}$ Aracati $(7,9 \%)$ e em Fortaleza (4,9\%), cidades do Ceará. Mesmo que não seja o objetivo principal deste estudo, é importante apontar o problema e investigar a sua associação com infecção genital e outros problemas de saúde em mulheres que buscam serviços de atenção ginecológica.

As limitações deste trabalho incluem o tamanho modesto e a natureza única da amostra (uma unidade do PSF), o que poderia limitar as inferências a respeito de outras mulheres em condições similares. No entanto, o alto índice de resposta tem uma força que poderia, de certa forma, diminuir essa potencial limitação. A possibilidade de respostas direcionadas, pela tendência à produção de respostas socialmente aceitáveis, não deve ser ignorada. Podem ter ocorrido imprecisões ao lembrar do uso do preservativo, idade na primeira relação sexual e número de parceiros sexuais. No entanto, o índice de participação demonstra que os programas de prevenção a IST/HIV podem implementar, com sucesso, serviços confidenciais para as mulheres atendidas pelo PSF.

Este estudo chama atenção para a importância da vigilância da saúde da mulher e aponta algumas maneiras pelas quais a saúde reprodutiva pode ser documentada, com o objetivo de melhorar as intervenções na atenção às infecções ginecológicas. O PSF é uma estratégia importante para o alcance de melhores métodos de prevenção e assistência, porque ele possibilita acesso mais precoce dessa população à saúde. Os índices preponderantes de infecções genitais neste estudo mostram que há um problema que deve ser controlado e uma necessidade de ação direcionada à avaliação de comportamentos de risco, visando, dessa forma, a prevenção de infecção genital.

\section{Agradecimentos}

Os autores gostariam de agradecer a Roche Diagnóstica Brasil pela doação dos testes para diagnóstico da infecção pela Chlamydia trachomatis (Amplicor Roche).

\section{Referências}

1. World Health Organization (WHO). Global prevalence and incidence of selected curable sexually transmitted infections: overview and estimates. Geneva: WHO; 2001.

2. Over $M$, Piot P. Human immunodeficiency virus infection and other sexually transmitted diseases in developing countries: public health importance and priorities for resource allocation. J Infect Dis. 1996;174 Suppl 2:S162-75.
3. Cates W Jr, Rolfs RT Jr, Aral SO. Sexually transmitted diseases, pelvic inflammatory disease, and infertility: an epidemiologic update. Epidemiol Rev. 1990;12:199-220.

4. Wasserheit JN. Epidemiological synergy. Interrelationships between human immunodeficiency virus infection and other sexually transmitted diseases. Sex Transm Dis. 1992;19(2): 61-77. 
5. Cohen MS. Sexually transmitted diseases enhance HIV transmission: no longer a hypothesis. Lancet. 1998;351 Suppl 3:5-7.

6. Sewankambo N, Gray RH, Wawer M, Paxton L, McNaim $D$, Wabwire-Mangen $F$, et al. HIV-1 infection associated with abnormal vaginal flora morphology and bacterial vaginosis. Lancet. 1997;350(9077): 546-50.

7. Oliveira FA, Pfleger V, Lang K, Heukelbach J, Miralles I, Fraga $F$, et al.Sexually transmitted infections, bacterial vaginosis, and candidiasis in women of reproductive age in rural Northeast Brazil: a population-based study. Mem Inst Oswaldo Cruz. 2007; 102(6):751-6.

8. Ministério da Saúde. Secretaria de Assistência à Saúde. Departamento de Atenção Básica (DAB). Evolução do credenciamento e implantação da estratégia Saúde da Família [homepage da Internet]. Brasília; 2004 [citado 11 abr 2008]. Disponível em: http://dtr2004. saude.gov.br/dab/localiza_cadastro.php

9. Brasil. Ministério da Saúde. Secretaria de Vigilância em Saúde. Programa Nacional de DST e Aids. Manual de controle das doenças sexualmente transmissíveis. Brasília: Ministério da Saúde; 2006.

10. Solomon D, Davey D, Kurman R, Moriarty A, O'Connor D, Prey $M$, et al. The 2001 Bethesda System: terminology for reporting results of cervical cytology. JAMA. 2002;287(16):21 14-9.

11. Amsel R, Totten PA, Spiegel CA, Chen KC, Eschenbach D, Holmes KK. Nonspecific vaginitis.Diagnostic criteria and microbial and epidemiologic associations.Am J Med. 1983;74(1):14-22.

12. Melles HH, Colombo S, Linhares IM, Siqueira LF. Avaliação de parâmetros para o diagnóstico laboratorial de infecção genital feminina por Chlamydia trachomatis. Rev Soc Bras Med Trop. 2000;33(4):355-61.

13. Bastos Fl, Lowndes CM, Castello-Branco LR, Linhares-de-Carvalho MI, Oelemann W, Bernier F, et al. Sexual behaviour and infection rates for HIV, blood-borne and sexually transmitted infections among patients attending drug treatment centres in Rio de Janeiro, Brazil. Int J STD AIDS. 2000; 11 (6):383-92.

14. Codes JS, Cohen DA, Melo NA, Santos AB, Codes JJ, Silva Júnior $J C$, et al. Detecção de doenças sexualmente transmissíveis em clínica de planejamento familiar da rede pública no Brasil. Rev Bras Ginecol Obstet. 2002;24(2):101-6.

15. Martins TA, Bello PY, Pontes LRSK, Costa LV, Miralles IS, Queiroz TRBS. As doenças sexualmente transmissíveis são problemas entre gestantes no Cearáe DST J Bras Doenças Sex Transm. 2004; 16(3):50-8.

16. Miranda AE, Szwarcwald CL, Peres RL, Page-Shafer K. Prevalence and risk behaviors for chlamydial infection in a populationbased study of female adolescents in Brazil. Sex Transm Dis. 2004;31(9):542-6.

17. Gonçalves AA, Paes e Alcântara MF, Silva LA. Incidência de gonorréia em população previdenciária da Baixada Santista. Rev Bras Patol Clin. 1984;20(2):47-52.

18. Kent HL. Epidemiology of vaginitis. Am J Obstet Gynecol. $1991 ; 165(4 \mathrm{Pt} 2): 1168-76$.
19. Adad SJ, Lima RV, Sawan ZT, Silva ML, Souza MA, Saldanha JC, et al. Frequency of Trichomonas vaginalis, Candida sp and Gardnerella vaginalis in cervical-vaginal smears in four different decades. São Paulo Med J. 2001;1 19(6):200-5.

20. Lourenço NCV, Sordi CR, Alvarenga VLS, Cicarelli RMB. Use of $P C R$ reactions (polymerase chain reaction) on Trichomonas vaginalis diagnosis in patients from Araraquara region (State of São Paulo, Brazil). Rev Cienc Farm. 2001 ;22(2):307-18.

21. Simões-Barbosa A, Feijó GC, Silva JX, Leal IIR, Barbosa TWP. Sixyear follow-up survey of sexually transmitted diseases in Brasilia, the capital of Brazil. Braz J Infect Dis. 2002;6(3):1 10-8.

22. Murta EF, Souza MA, Araújo Júnior E, Adad SJ. Incidence of Gardnerella vaginalis, Candida sp and human papilloma virus in cytological smears. Sao Paulo Med J. 2000; 1 18(4): 105-8.

23. Nonnenmacher B, Breitenbach V, Villa LL, Prolla JC, Bozzetti MC. Identificação do papilomavírus humano por biologia molecular em mulheres assintomáticas. Rev Saude Publica. 2002;36(1):95-100.

24. Franco EL, Villa LL, Ruiz A, Costa MC. Transmission of cervical human papillomavirus infection by sexual activity: differences between low and high oncogenic risk types. J Infect Dis. 1995;172(3): 756-63.

25. Rama CH, Roteli-Martins CM, Derchain SFM, Longatto-Filho A, Gontijo RC, Sarian LOZ, et al. Prevalência do HPV em mulheres rastreadas para o câncer cervical. Rev Saude Publica. 2008;42(1):123-30.

26. Praça NS, Latorre MRDO, Hearst N. Fatores associados à percepção de risco de infecção pelo HIV por puérperas internadas. Rev Saude Publica. 2003;37(5):543-51.

27. Mpairwe H, Muhangi L, Namujiu PB, Kisitu A, Tumusiime A, Muwanga $M$, et al. HIV risk perception and prevalence in a program for prevention of mother-to-child HIV transmission: comparison of women who accept voluntary counseling and testing and those tested anonymously. J Acquir Immune Defic Syndr. 2005;39(3):354-8.

28. Maharaj P, Cleland J. Risk perception and condom use among married or cohabiting couples in KwaZulu-Natal, South Africa. Int Fam Plan Perspect. 2005;31 (1):24-9.

29. Chirinos JL, Bardales O, Segura MD. Las relaciones coitales y la percepción de riesgo de adquirir ETS/SIDA en adultos jóvenes varones de Lima, Perú. Cad Saude Publica. 2006;22(1):79-85.

30. Yin YP, Wu Z, Lin C, Guan J, Wen Y, Li L, et al. Syndromic and laboratory diagnosis of sexually transmitted infection: a comparative study in China. Int J STD AIDS. 2008;19(6):381-4.

31. Lush L, Walt G, Ogden J. Transferring policies for treating sexually transmitted infections: what's wrong with global guidelines? Health Policy Plan. 2003; 18(1):18-30.

32. World Health Organization (WHO). Guidelines for the management of sexually transmitted infections. Geneva: WHO; 2001.

33. Mayaud P, ka-Gina G, Cornelissen J, Todd J, Kaatano G, West B, et al. Validation of a WHO algorithm with risk assessment for the clinical management of vaginal discharge in Mwanza, Tanzania. Sex Transm Infect. 1998;74 Suppl 1:S77-84. 